\title{
Descripción del uso tradicional de plantas medicinales en mercados populares de Bogotá, D.C.
}

\author{
Traditional use of medicinal plants in markets from Bogotá, D.C.
}

Sara Emilia Giraldo Quintero, María Consuelo Bernal Lizarazú, Adriana Morales Robayo, Alesdy Zuled Pardo Lobo, Leopoldo Gamba Molano.

\section{Resumen}

Objetivo. Describir el uso tradicional de 26 plantas, identificando la droga vegetal, su preparación y administración, los fines medicinales para los que se recomiendan y posibles prácticas de uso inadecuado. Materiales y métodos. Se aplicaron 16 entrevistas semiestructuradas a vendedores de plantas medicinales en 8 plazas de mercado de la ciudad de Bogotá, D.C. Adicionalmente se realizó la identificación taxonómica de las plantas con mayor reporte por parte de los vendedores y su uso tradicional fue comparado con las indicaciones farmacológicas reportadas en el Vademecum Colombiano de Plantas Medicinales. Resultados. Las plantas de mayor citación entre los vendedores entrevistados fueron: "Cidrón", "caléndula" y "manzanilla", seguidas por "cola de caballo", "ruda" y "albahaca" las cuales presentaron al menos tres reportes cada una. Se encontró que algunas de las plantas mencionadas por los vendedores no se reportan en el Vademécum Colombiano de Plantas Medicinales, entre ellas "paico", "chitato", "alfalfa", "laurel" y "suelda consuelda". Se detectó poco conocimiento por parte de los vendedores, sobre las posibles contraindicaciones o efectos adversos de las plantas medicinales lo que conlleva a prácticas inadecuadas en la comunidad. También se evidenció desconocimiento de las diferencias entre las formas de preparación infusión y decocción. Se identificaron plantas medicinales que pueden ser promisorias terapéuticamente por nuevos usos reportados, entre ellas "albahaca", "caléndula", "cidrón", "cola de caballo" y "manzanilla".

Palabras clave: plantas medicinales, etbotánica, etnofarmacología, medicina tradicional, fitoterapia, mercados populares.

\begin{abstract}
Objective: To describe information about traditional use of 26 plants, identifying plant drug, preparation and administration, medicinal purposes for recommended practices and possible misuse. Materials and Methods. This work was performed in 8 popular markets of Bogotá. By applying semi-structured interviews with 16 sellers of medicinal plants. Further taxonomic identification of
\end{abstract}


medicinal plants was carried out with greater reporting by sellers and their traditional use was compared with pharmacological indications reported in the Vademecum Colombiano de Plantas Medicinales. Results. Report higher plants between sellers respondents were "cidrón", "calendula" and "manzanilla", followed by "cola de caballo", "ruda" and "albahaca", which presented at least three reports each one. It was found that some of the plants mentioned by the sellers do not appear in the Vademecum Colombiano de Plantas Medicinales plants including "paico", "chitato", "alfalfa", "laurel" and "suelda consuelda". Little knowledge was detected by sellers about possible contraindications or adverse effects of medicinal plants which leads to inadequate practices within the community. Ignorance of the differences between the forms of decoction and infusion preparation was also evidenced. Plants that may be promising therapeutic for new uses reported were identified including "albahaca”, "calendula", "cidrón”, "cola de caballo" and "manzanilla".

Keywords: medicinal plants, ethnobotany, ethnopharmacology, traditional medicine, phytotherapy, herbal markets.

\section{Introducción}

Según la Declaración de Alma-Ata (1) y la Declaración de Beijing, adoptada en el Congreso de la OMS sobre Medicina Tradicional (2), esta práctica es un componente esencial dentro de la atención primaria en salud, especialmente en países en vía de desarrollo. La Declaración de Beijing también manifiesta "la necesidad de que la comunidad internacional, los gobiernos, los profesionales y trabajadores sanitarios actúen y cooperen para velar por que la medicina tradicional se utilice adecuadamente como elemento importante que contribuye a la salud de todas las personas".

Colombia es considerada un país privilegiado por ser reconocido como el segundo en el ámbito mundial en diversidad de especies vegetales, adicionalmente al menos 6.000 de estas especies poseen propiedades medicinales. El uso tradicional de plantas medicinales es ampliamente reconocido en los mercados populares, donde plantas silvestres se emplean para diversidad dolencias (3).

En Bogotá, D.C, la comercialización de las plantas medicinales se lleva cabo ampliamente en los mercados distritales de la ciudad, soportado por un conocimiento etnobotánico que sobre medicina tradicional ha trascendido de generación en generación y que vale la pena dar valor. En las plazas de mercado además de comercializarse una gran cantidad de especies nativas que podrían contribuir a la investigación y desarrollo de productos fitoterapéuticos, la creencia por parte de la comunidad de no ser tóxicas contribuye a un uso inadecuado de las mismas.

Algunos estudios etnobotánicos orientados al inventario y descripción del uso tradicional de plantas medicinales en mercados locales en diferentes países, son los realizados por Macía et al (4), de Albuquerque et al (5), Giraldo et al (6), Mati y Boer (7), Boudjelal et al (8), Leitãoa et al (9) y Quiroz et al (10).

Dentro de los estudios sobre plantas medicinales realizados en mercados populares (plazas de mercado) en Colombia, se resalta el de Guerrero y Támara (11), el cual tiene una orientación etnofarmacológica, en el cual se recopiló información sobre la comercialización y uso de quince plantas medicinales en tres mercados populares de Bogotá, comparando el uso tradicional informado con estudios farmacológicos y toxicológicos reportados en diferentes fuentes bibliográficas. 
Adicionalmente los resultados encontrados se publicaron en una cartilla didáctica para la comunidad.

De la misma forma, son pertinentes los realizados por Mesa y Linares (12) y Valderrama y Linares (13), en los cuales se explora el uso de fracciones, frutos y semillas de plantas medicinales en siete plazas de mercado de Bogotá, reconociendo que aunque en estos escenarios se comercializan una gran cantidad de plantas, no se tiene total certeza sobre los usos medicinales recomendados y no necesariamente los nombres populares de las mismas corresponden a la especie vegetal comercializada, de tal manera que la posible desinformación transferida por los vendedores podría conllevar problemas de salud entre los usuarios.

El presente trabajo describe el uso tradicional de plantas medicinales a partir de los saberes y prácticas en ocho plazas de mercado de la ciudad de Bogotá, con el fin de identificar plantas promisorias por su potencial terapéutico y adicionalmente evidenciar el posible uso irracional que se podría estar generando en la comunidad.

\section{Materiales y métodos}

El presente estudio se llevó a cabo en mercados populares (plazas de mercado) de Bogotá, D.C. Las cuales presentan puestos de venta de plantas medicinales en su comercio. Se visitaron siete plazas de mercado distritales de Bogotá (Santander, Las Ferias, el Carmen, La Concordia, San Benito, Trinidad Galán y San Carlos). Se incluyó además la Plaza de Mercado de Paloquemao, que no es Distrital, pero si es una de las principales plazas de acopio y distribución mayorista de plantas medicinales de la ciudad. Las plazas de mercado a visitar fueron asignadas al azar.

El estudio siguió una metodología descriptiva de corte transversal y participativa. La comunidad de estudio fueron informantes (vendedores), conocedores de plantas medicinales en cada plaza de mercado visitada, seleccionados según los siguientes criterios de inclusión: (i) vendedores de plantas medicinales en puestos de venta en cada plaza de mercado, (ii) respuesta positiva para participar y responder las preguntas de la entrevista, (iii) mayores de 18 ańos.

La selección de las plantas medicinales se realizó empleando el método etnofarmacológico de muestreo recomendado por Martin (14). La recopilación de la información sobre el uso tradicional de plantas medicinales se hizo mediante entrevista semiestructurada aplicada a los vendedores entre los meses de Julio a Octubre de 2012, la cual fue diseńada y aplicada de acuerdo a las recomendaciones de Martin para estudios etnobotánicos realizados en mercados locales (14). Se consultaron y midieron variables sociodemográficas y conocimientos sobre prácticas relacionadas con el uso tradicional de plantas medicinales. Los resultados obtenidos fueron analizados comparándolos con la información reportada en el Vademécum Colombiano de Plantas Medicinales (15).

De acuerdo a la frecuencia de citación de cada planta, se seleccionaron algunas para su determinación taxonómica. La determinación taxonómica de las plantas, fue realizada con el apoyo del Herbario de Plantas Útiles de Colombia (HPUJ) de la Pontificia Universidad Javeriana, siendo posible identificar género y especie de cada planta.

\section{Resultados}

En las ocho plazas de mercado visitadas se encontró un total de 43 puestos de venta de plantas medicinales, no todos los vendedores accedieron a brindar información. Muchos de ellos se notaban prevenidos para hacerlo bien sea por desconfianza o sencillamente argumentaban falta de tiempo. Por lo anterior solamente 16 vendedores colaboraron para responder a la entrevista propuesta para el desarrollo del presente trabajo así: Plaza de Mercado Distrital Las Ferias y Plaza de Mercado de Paloquemao, cinco vendedores en cada una, para el resto de plazas de mercado distritales visitadas, un vendedor en cada una. 
La edad promedio de los vendedores es de 53 años. La persona más joven tiene 27 años, 8 personas tienen entre 51 y 59 ańos y los de mayor edad tienen 65 años. De los 16 vendedores entrevistados, solamente dos personas alcanzaron el nivel tecnológico, nueve personas alcanzaron únicamente la educación primaria y tres personas no tiene nivel educativo. Los conocimientos tradicionales sobre plantas medicinales los adquirieron por autoaprendizaje (diez vendedores), por enseñanzas de los padres (cinco vendedores) o por enseńanzas de los abuelos (un vendedor). Doce personas de las dieciséis entrevistadas están cubiertos por el SISBEN en nivel 1 y 2 , solo uno de ellos está cubierto por el POS y tres personas no cuenta con servicio de salud. Catorce de los vendedores se dedica exclusivamente a este oficio en puestos permanentes y solamente dos personas, además de ser vendedores de plantas en puestos temporales ejercen como comerciantes u oficios varios $\mathrm{y}$ son quienes alcanzaron un mayor nivel educativo. De las personas entrevistadas doce de ellas son solo vendedores y cuatro son vendedores recolectores, que tienen entre 9 y 40 ańos de ejercer este oficio.

Sobre el uso tradicional de las plantas medicinales en las plazas de mercado visitadas, las plantas de mayor frecuencia de citación entre los vendedores entrevistados fueron: "Cidrón", "caléndula" y "manzanilla" con cuatro reportes cada una, seguidas por "cola de caballo", "ruda" y "albahaca" las cuales presentaron tres citaciones. Plantas como "hierbabuena", "ajenjo", "llantén”, "toronjil”, "chitato", "sauco", "anamú" y "ortiga” fueron citadas dos veces por los vendedores, las demás plantas fueron citadas una vez, Figura 1.

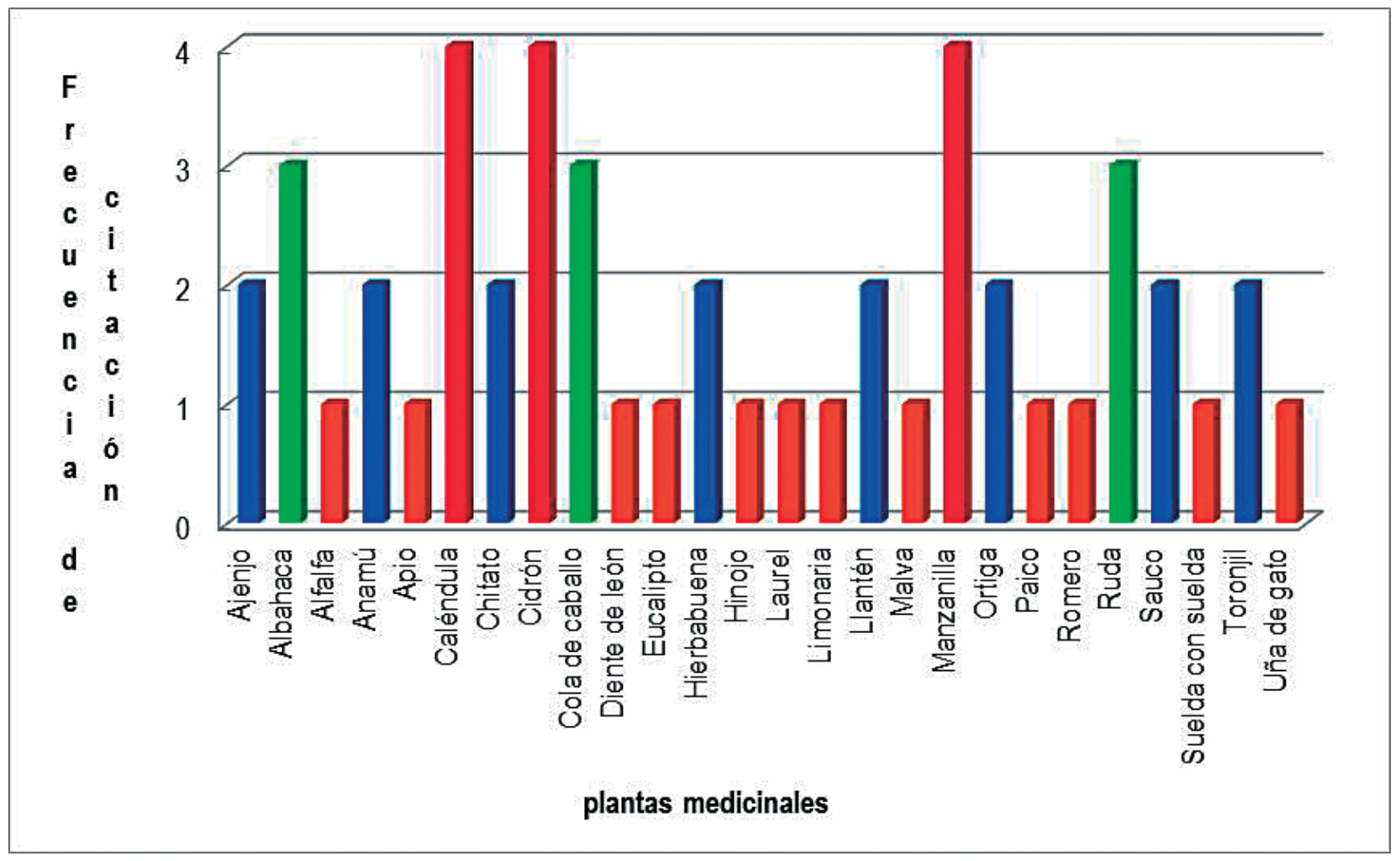

Figura 1. Frecuencia de citación de plantas medicinales mencionadas por los vendedores en las plazas de mercado visitadas 
En la Tabla 1 se presentan los resultados sobre el uso tradicional de las plantas medicinales con mayor citación entre los vendedores entrevistados (entre tres y cuatro reportes), en comparación con la indicación farmacológica reportada en el Vademecum Colombiano de Plantas Medicinales (VCPM).

Tabla 1. Uso tradicional de plantas medicinales reportadas por los vendedores entrevistados y comparación con el uso indicado en el Vademécum Colombiano de Plantas Medicinales

ACTIVIDADES

PELIGROSAS
Forma de preparación

Droga vegetal y Uso tradicional
Droga vegetal e Indicación farmacológica (VCPM)

Planta completa: Uso interno: anti flatulento, coadyuvante en el tratamiento de la distención abdominal y dolores abdominales.

Albahaca

Ocimum americanum Linneo

Infusión o decocción estómago, para el colon, cálculos renales y como aromática.

Planta entera; tallos, hojas y flores: Inflamaciones, dolor de oído, para golpes, quemaduras por el sol, cirugías e hinchazones, gastritis, úlcera gástrica, úlceras de piel, inflamación del cólon, quemaduras, para los pies o manos agrietadas.

Hojas: Problemas digestivos, para mejorar la circulación de la sangre, dolor de muela, estrés, tranquilizante y para el corazón.
Cidrón

Aloysia citriodora Palau

En infusión, solo o con toronjil y valeriana
Flores: Uso interno: antiinflamatorio, tratamiento de alteraciones inflamatorias bucofaríngeas. Uso externo: Antiinflamatorio, cicatrizante, tratamiento tópico de inflamaciones de la piel y mucosas.
Hojas: Uso interno: anti flatulento, sedante, coadyuvante en el tratamiento de dispepsias y espasmos gastrointestinales.

\section{Cola de caballo}

Equisetum bogotense Kunth
Planta entera: Limpia el sistema urinario, expulsa cálculos renales, sirve como diurético.
Partes aéreas: Uso interno: diurético.

\section{Manzanilla}

Matricaria chamomilla Linneo
Infusión y decocción; macerar para emplastos estomacales
Planta entera; tallos y flores: Dolor de estómago, caída del cabello, dolor menstrual, reacciones alérgicas, cicatrices, estrés, gases, dolores articulares, dolores de cabeza, relajante muscular, y tranquilizante.
Sumidades floridas: Uso interno: anti inflamatorio, antiespasmódico, coadyuvante en el tratamiento sintomático de trastornos digestivos. Uso externo: Anti inflamatorio, coadyuvante en el tratamiento de inflamaciones e irritaciones de la piel y mucosas.

Partes aéreas: Uso interno Emenagogo.

\section{Ruda}

Ruta graveolens Linneo
Picada y mezclada con huevo cocido; decocción
Tallos, hojas y flores: Nutre la matriz, inflamación de la matriz. 
De las plantas mencionadas por los vendedores algunas no aparecen en el VCPM entre ellas: "Paico" ("purgante"), "chitato" (para la "mala circulación"), "alfalfa" (para "hipertensión arterial", para "hemorragias"), "laurel" (para "infección de heridas") y "suelda consuelda" (para "inflamaciones externas y luxaciones"). El VCPM es el documento oficial que contiene la información general sobre las plantas medicinales aceptadas en el país para ser utilizadas en productos fitoterapéuticos (15). Las plantas medicinales no incluidas merecen la realización de estudios farmacológicos y toxicológicos suficientes que sustenten sus usos tradicionales. Las plantas medicinales de mayor citación fueron identificadas taxonómicamente confirmando género y especie. Para la mayoría de las plantas medicinales los vendedores no mencionaron recomendaciones o precauciones durante su uso. Para el caso de "cola de caballo" (Equisetum bogotense Kunth), manifiestan como precaución que se debe tomar solamente por ocho días porque "acorta la vista".

\section{Discusión}

De acuerdo a los resultados obtenidos se evidencia la importancia que sigue teniendo el ejercicio de las prácticas de la medicina tradicional en nuestras comunidades, resaltándose por vendedores que se dedican exclusivamente a este oficio, cuyo saber etnobotánico lo han adquirido la gran mayoría por autoaprendizaje. Es preocupante que aún haya personas sin protección social evidenciándose la desatención del estado en materia de salud en estas comunidades.

Con respecto al uso tradicional, aunque para la mayoría de las plantas medicinales los vendedores no mencionaron recomendaciones o precauciones durante su uso, cabe resaltar que en el VCPM (15) se consideran contraindicaciones y precauciones, como lo establecido para el "ajenjo" que está contraindicado en caso de epilepsia y embarazo y puede presentar síntomas de toxicidad por uso prolongado. Caso similar sucede con "anamú”, "caléndula" (Calendula officinalis Linneo), "diente de león", "eucalipto", "malva”, "ortiga”, "romero", "ruda” (Ruta graveolens Linneo), "sauco” y uňa de gato" que están contraindicadas en embarazo y lactancia; la "ruda" además interactúa con medicamentos antihipertensivos por lo que está contraindicada en personas que reciban estos medicamentos o que tengan historia de insuficiencia cardiaca o arritmias.

El uso del cidrón (Aloysia citriodora Palau) y manzanilla (Matricaria chamomilla Linneo), debe ser con precaución por hipersensibilidad a cualquiera de sus componentes. Los vendedores para el caso de "cola de caballo" (Equisetum bogotense Kunth), manifiestan no tomarla por uso prolongado porque "acorta la vista", sin embargo en el VCPM no se menciona ninguna contraindicación o precaución para esta planta. Es necesario profundizar en estudios de toxicidad vigentes sobre esta planta, considerando que según lo reporta el VCPM no se han apreciado signos clínicos de toxicidad.

Al comparar la información proporcionada por los vendedores sobre el uso tradicional de las plantas medicinales, con las indicaciones allí reportadas en el VCPM, se encuentra que para las de mayor citación coinciden con al menos una de las dolencias para las cuales están indicadas. Sin embargo, los vendedores mencionan además otros usos no reconocidos en el VCPM, que pueden ser promisorios por sus utilidades terapéuticas, entre ellos: Albahaca (Ocimum americanum Linneo): para el colon y cálculos renales; caléndula (Calendula officinalis Linneo): gastritis y úlcera gástrica; cidrón (Aloysia citriodora Palau): para mejorar la circulación y para el corazón; cola de caballo (Equisetum bogotense Kunth): para expulsar cálculos renales; manzanilla (Matricaria chamomilla Linneo): dolores de cabeza, articulares y tranquilizante.

Es importante resaltar el uso que los vendedores confieren a la ruda (Ruta graveolens Linneo), que podría estar relacionado con el uso reconocido en el VCPM (emenagogo), sin embargo desconocen 
su contraindicación en embarazo y lactancia. La infusión y la decocción son las formas de uso tradicional más recomendado entre los vendedores. Durante la entrevista fué notorio que no tienen clara la diferencia entre éstas dos formas de obtención del extracto acuoso. Para el caso de la caléndula (Calendula officinalis Linneo) y la manzanilla (Matricaria chamomilla Linneo), los vendedores confieren propiedades medicinales también a la planta entera o a otras partes como hojas y tallos, sin embargo el VCPM sólo reconoce las flores, como la droga vegetal de estas plantas. Por lo anterior es necesaria la revisión de estudios fitoquímicos que den validez al uso medicinal de otras fracciones de estas plantas por su presencia de principios activos.

Para Bermúdez et al (16), una investigación etnobotánica que esté enfocada en el estudio de las plantas medicinales implica la realización de un proyecto interdisciplinario que constaría de las siguientes fases: (i) la documentación del conocimiento tradicional, (ii) la determinación cuantitativa de resultados, (iii) la evaluación fitoquímica, farmacológica y toxicológica y (iv) el desarrollo de mecanismos para compensación a la comunidad, planteando dentro de estos últimos, los siguientes: diseñar proyectos de aprovechamiento sostenible o estrategias de conservación de los recursos y los ecosistemas naturales; desarrollar mecanismos para el reconocimiento público de los derechos intelectuales sobre el conocimiento tradicional y desarrollar estrategias para compensar a la población de las comunidades por su participación en las investigaciones.

Dentro de las estrategias educativas para la publicación de los resultados de estudios etnobotánicos y recuperación del conocimiento tradicional en las comunidades, Martin (14) menciona entre otros boletines, publicaciones populares, exposiciones y talleres.

Los resultados encontrados en el presente trabajo, evidencian la necesidad de la implementación de programas educativos y estrategias que promuevan el uso adecuado de las plantas medicinales, profundizando especialmente en las precauciones, contraindicaciones, interacciones medicamentosas y efectos adversos que un uso inadecuado o irracional de las mismas pueda estar generando en nuestras comunidades.

\section{Agradecimientos}

Los autores agradecen al Sistema de Gestión de Investigación (SIGI) de la UNAD, por la financiación del proyecto "Uso tradicional de plantas medicinales y promoción de su uso adecuado en mercados populares de Bogotá, D.C.” (SIGI 0040, Convocatoria 002) y al Herbario de Plantas Útiles de Colombia (HPUJ) de la Pontificia Universidad Javeriana por la determinación taxonómica de las plantas medicinales.

\section{Referencias}

1. OMS. Alma-Ata 1978 Atención Primaria en Salud. Ginebra, Suiza: OMS; 1978.

2. Declaración de Beijing. En: Congreso de la OMS sobre Medicina Tradicional. Beijing, China; 2008.

3. Proexport. Estudio de Oferta y Demanda del Sector de Productos Naturales, Programa de Promoción del Comercio Sur-Sur (CCI). Bogotá, Colombia: Centro de Comercio Internacional UNCTAD/OMC; 2003.

4. Macía MJ, García E, Vidaurre PJ. An ethnobotanical survey of medicinal plants commercialized in the markets of La Paz and El Alto, Bolivia. J Ethnopharmacol. 2005;97(2): $337-350$

5. Albuquerque UP, Monteiro JM, Ramos MA, de Amorim EL. Medicinal and magic plants from a public market in northeastern Brazil. J Ethnopharmacol. 2007;110(1): 7691.

6. Giraldo D, Baquero E, Bermúdez A, Oliveira MA. Caracterización del comercio de plantas medicinales en los mercados populares de Caracas, Venezuela. Acta Bot Venez 2009;32(2): 267-301.

7. Mati E, de Boer H. Ethnobotany and trade of medicinal plants in the Qaysari Market, Kurdish Autonomous Region, Iraq. J Ethnopharmacol. 2011;133(2): 490-510.

8. Boudjelal A, Henchiri Ch, Sari M, Sarri D, Hendel N, Benkhaled A, Ruberto G. Herbalists and wild medicinal plants in M'Sila (North Algeria): An ethnopharmacology survey. J Ethnopharmacol. 2013;148(2): 395-402. 
9. Leitãoa F, Leitãoa SG, da Fonseca-Kruelb VS, Silvac IM, Martinsa K. Medicinal plants traded in the open-air markets in the State of Rio de Janeiro, Brazil: an overview on their botanical diversity and toxicological potential. Rev Bras Farmacogn. 2014;24(2): 225-247.

10. Quiroz D, Towns A, Legba S, Swier J, Brière S, Sosef M, Andel T. Quantifying the domestic market inherbal medicine in Benin, West Africa. J Ethnopharmacol. 2014;151(3): 1100-1108.

11. Guerrero EL y Támara SM. Evaluación de los usos populares, información química, farmacológica y toxicológica de 15 especies medicinales vendidas en tres plazas de mercado de Bogotá. Colombia [tesis de grado]. Bogotá: Universidad Nacional de Colombia; 2005.

12. Mesa LI, Linares EL. Fracciones de plantas medicinales vendidas en plazas de mercado de Bogotá, Colombia. En:
Acta Biol Colomb: V Encuentro Científico de Estudiantes de Biología. Bogotá; 2006;11(1): 151-152.

13. Valderrama NM y Linares EL. Frutos y semillas medicinales vendidas en plazas de mercado de Bogotá, Colombia. En: Acta Biol Colomb: V Encuentro Científico de Estudiantes de Biología. Bogotá; 2006;11(1): 145-146.

14. Martin GJ. Etnobotánica, Manual de Métodos: Volumen. 1. Montevideo, Uruguay: Nordan-Comunidad; 2001.

15. Ministerio de la Protección Social. Vademecum Colombiano de Plantas Medicinales. Bogotá, Colombia: Arte y Sistemas Integrados; 2008.

16. Bermúdez A, Oliveira-Miranda MA, Velázquez D. La investigación etnobotánica sobre plantas medicinales: Una revisión de sus objetivos y enfoques actuales. Interciencia. 2005;30(8): 453-459. 